\title{
RHESSI and TRACE Observations for the Impulsive Flare Loops
}

\author{
W. Q. Gan and Y. P. Li \\ Purple Mountain Observatory, Chinese Academy of Sciences, Nanjing 210008, China \\ email: wqgan@pmo.ac.cn
}

\begin{abstract}
After summarizing the geometric relationship of the RHESSI flare X-ray sources with the TRACE flare loops, and the motion modes of RHESSI flare X-ray sources, we concentrate on the newly discovered downward motion of flare looptop X-ray source. In particular, we present a new argument for the flare loop shrinkage during the impulsive phase: TRACE $195 \AA$ loop shrinkage. The temporal behavior of $195 \AA$ loop shrinkage and its magnitude are consistent with that revealed from the RHESSI X-ray sources. Further study shows that the TRACE $195 \AA$ loop shrinkage is in the form of oscillation, with a period of about $150 \mathrm{~s}$ and an amplitude of about $300 \mathrm{~km}$. We discuss this oscillatory shrinkage with respect to a numerical simulation of reconnection model.
\end{abstract}

Keywords. Sun: flares, Sun: X-rays, gamma-rays, Sun: UV radiation

\section{Introduction}

Most of recent progress on high energy solar phenomena are due to the success of RHESSI, which was launched on 2002 February 5 and is still working well in the orbit. The detailed introduction about the RHESSI mission can be found in Lin et al. (2002). The primary scientific objectives of RHESSI is to explore the basic physics of particle acceleration and energy release in solar flares. From the observations for more than four years, the RHESSI has fully fulfilled its primary scientific objectives.

To summary all the results obtained with RHESSI is a hard work, but the readers can refer to the recent reviews by Lin (2004), Smith (2004), Krucker \& Hudson (2004), Share \& Murphy (2004, 2005), Dennis et al. (2005), and Gan (2006). In this paper I just review in brief the morphology of X-ray sources and their motions, together with the supplementary observations made by TRACE. Then we focus on the shrinkage motion of flare loops during the impulsive phase. Finally we present a new work of so-called oscillatory shrinkage of impulsive flare loops and discuss its significance.

\section{Classifications of Hard X-ray Sources}

Based on RHESSI image observations, the X-ray sources could be classified into four kinds: footpoint sources; looptop sources; coronal sources; and coronal loop sources. Figure 1 shows an example of hard X-ray images at the maximum moment, superposed on the post flare loops seen in TRACE $195 \AA$ for the X4.8 flare of 2002 July 23 (Li \& Gan 2005a). The X-ray image at $28-38 \mathrm{keV}$ seems to be able to reflect all of these different sources. Figure 2 shows another example for the M7.6 flare on 2003 October 24 (Gan et al. 2004), where the Nobeyama radio loop at $34 \mathrm{GHz}$ is compared with RHESSI images and TRACE $1600 \AA$ image.

For the two footpoint sources, they are usually in a conjugate form, that is, they have similar temporal and nonthermal spectral properties. For the looptop source, it resembles 
so-called Masuda flare discovered first from Yohkoh observations (Masuda et al. 1994). This source, in nonthermal form, appears at the top or a little above the SXR loop. The footpoint sources and looptop source altogether constitute a low-lying loop. While for the coronal source, it is a new kind discovered by RHESSI (Lin et al. 2003). This source, initially in nonthermal form, appears above the looptop source before the impulsive phase, when there is no chromospheric counterparts, like bright ribbons. Afterward, it may become a thermal or super hot source. Another new kind of hard X-ray source is the coronal loop source (Veronig et al. 2004), which presents a nonthermal emission. Veronig et al. (2004) explained this source as electron beams suffering a thick-target interactions in a dense loop.

Obviously, these X-ray sources reflect their internal connections and provide an essential constraint to a basic model of solar flares. In particular, Sui et al. $(2003,2004)$ found a new evidence for magnetic reconnection that the looptop source and the coronal source has oppositely directed temperature gradients, that is, the temperature of the looptop source increases with the altitude whereas the temperature of the coronal source decreases with altitude. This elaborate scenario means that the highest temperatures must be located in the regions just between the looptop source and the coronal source, where the magnetic reconnection happens. It was also thought an evidence for the existence of current sheet.

\section{Motion of Hard X-ray Sources}

The motion mode of hard X-ray sources is directly related to the magnetic reconnection. In the classic reconnection model (e.g., Priest \& Forbes 2002), the two hard X-ray footpoint sources should move apart as successive field lines are connected at higher altitude. However, observationally, the motion of hard X-ray footpoint sources can either decrease and increase in the separation across the neutral line, or parallel and antiparallel movements along the arcade, demonstrating how complex the flare is (Grigis \& Benz 2005 and reference in).

Krucker et al. (2003) studied the motion of hard X-ray footpoint sources for the X4.8 flare of 2002 July 23 and found that one footpoint source moves systematically for more than 10 minutes, but the other does not. Although the motion presents mostly in parallel rather than in separation, the good correlation between the speed of the motion and the hard X-ray light curve was thought a strong evidence for the magnetic reconnection model. The difference from the classic scenario of magnetic reconnection was explained as the complex magnetic configuration. However, no correlation between the speed of footpoint motion and hard X-ray flux were also observed recently (Grigis \& Benz 2005), suggesting that the hard X-ray light curve is caused by a spatial displacement along the arcade, that is, a disturbance propagates along the arcade, sequentially triggering a reconnection process in successive loops of the arcade.

Most recently, based on HXT/Yohkoh data Bogachev et al. (2005) classified the footpoint motions into three types. Type I consists of the motions preferentially away from and nearly perpendicular to the neutral line. Type II manifests the motions mainly along the neutral line in antiparallel directions. Type III is similar to Type II but all the HXR sources move in the same direction along the neutral line. The proportion of Type I, II, and III is $13 \%, 26 \%$, and $35 \%$, respectively. We see that the traditional two dimensional reconnection model can directly explain only a small number of flares (Type I). Bogachev et al. (2005) showed a carton to explain the motions of Type II, i.e., the magnetic line reconnection happens in a highly sheared magnetic configuration, while the formation of these sheared magnetic structures may result from large-scale photospheric flows like 

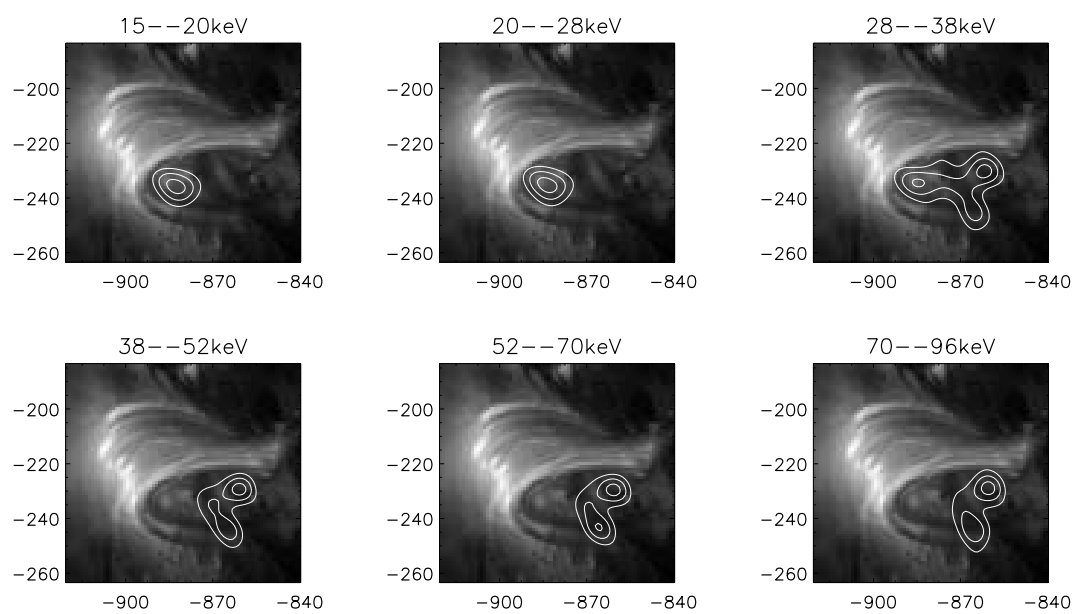

Figure 1. TRACE $195 \AA$ image (01:58:08) and RHESSI clean contour (00:28:36) (contour levels: $50 \%, 70 \%$, and $90 \%$ ) for the X4.8 flare on 2002 July 23.

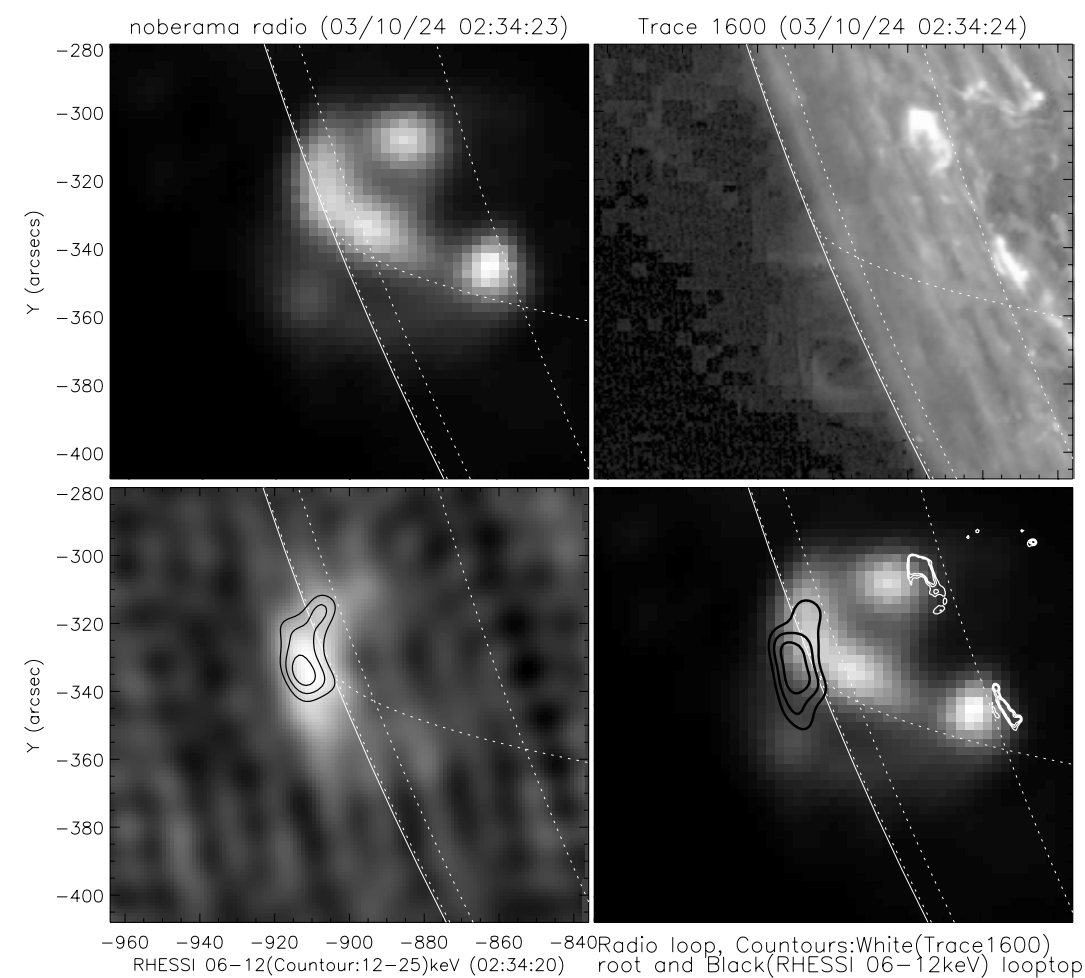

Figure 2. Nobeyama radio image at $34 \mathrm{GHz}$, TRACE image at $1600 \AA$, RHESSI images at 6-12 and $12-25 \mathrm{keV}$, and a comparison among these images for the M7.6 flare on 2003 October 24.

differential rotation or sheared vortex. For the motions of Type III, it was explained as a displacement of the particle acceleration region during a flare.

A downward motion of looptop hard X-ray source during the early impulsive phase was discovered by Sui et al. (2003, 2004). For the three flares studied, they found that within 2-4 minutes of the impulsive phase, the looptop sources in the X-ray 6-12 and 12-25 keV 
images first descended by about $20 \%$ with a velocity of about $10 \mathrm{~km} \mathrm{~s}^{-1}$ and then rose. Recently, Li \& Gan (2005b) confirmed this shrinkage motion through directly measuring the flare radio loop. They showed that in the impulsive phase the loop in the radio image at $34 \mathrm{GHz}$ shrank by about 30\%, suggesting a downward speed of approximately 13 $\mathrm{kms}^{-1}$. Besides, more and more RHESSI events with an apparent altitude decrease of looptop X-ray sources at the early impulsive phase were presented (Krucker et al. 2003; Liu et al. 2004; Veronig et al. 2006).

In the later time of impulsive phase, the upward motion of hard X-ray looptop source or the increase of loop height with time has been known before (e.g., Tsuneta et al., 1992). This motion was thought as another evidence for magnetic reconnection. The RHESSI observations give a more clarification of this kind of motion. The upward motion of hard $\mathrm{X}$-ray looptop source occurs after the peak of the hard X-ray flux. The upward motion, at a speed of $10-20 \mathrm{kms}^{-1}$, can be correlated to the hard X-ray light curve (Sui et al. 2004) or not (Liu et al. 2004).

For the motion of coronal hard X-ray source, the studies so far seem to be very limited. Sui et al. (2004) described for one flare that the coronal source stayed stationary for several minutes in the early impulsive phase. After the hard-X flux peak, the coronal source moved outward at a speed of $300 \mathrm{~km} \mathrm{~s}^{-1}$.

\section{Oscillatory Shrinkage of TRACE Loops}

As mentioned above, the downward motion of looptop X-ray sources is a new phenomenon discovered by RHESSI. The associated shrinkage of flare loops seen at $34 \mathrm{GHz}$ was presented by Li \& Gan (2005b). The importance of this discovery is that it is the first time to distinguish this kind of motion in respect to the explosive energy release during a flare. In particular, the previous flare models of magnetic reconnection have always been taken as explaining the apparent upward motions of flare loop system. Can these models explain this new kind of downward contraction? Besides, as a new kind of phenomenon, it is obviously necessary to confirm it from different observational points of view.

Recently, Li \& Gan (2006) identified this shrinkage motion from the TRACE observations for the M2.5 flare on 2002 April 16. Figure 3 presents the running difference images of TRACE $195 \AA$ (b-w reversed), overlaid on RHESSI contours at $60 \%$ and $90 \%$ of the peak intensity for $6-12$ and $12-25 \mathrm{keV}$ images.

From Figure 3 we see clearly that the TRACE $195 \AA$ loops in the running difference images shrink obviously. The middle panel of Figure 4 shows the altitudes of the TRACE $195 \AA$ looptop and the HXR looptop sources at $6-12 \mathrm{keV}$ and $12-25 \mathrm{keV}$ as a function of time during the shrinkage process. We omitted to show the later apparent upward motions seen at 6-12 and 12-25 keV sources, since a similar result has already been presented by Sui et al. (2004). The new result here is that in the rising phase of about 5 minutes the altitude of the TRACE $195 \AA$ loop in the running difference images shrank by about 30\%, suggesting a mean apparent downward speed of approximately $15.0 \mathrm{~km} \mathrm{~s}^{-1}$. This speed is very close to that deduced from the RHESSI 12-25 keV source. After 13:12 UT, the TRACE $195 \AA$ loop in the running difference images is hard to distinguish, implying the termination of the TRACE loop downward motion. This time is temporally consistent with that when the X-ray sources change to move upward.

Checking carefully the shrinkage of TRACE $195 \AA$ loop, we find an oscillatory component. We fit the observations with a combination of a sine wave and a linear background. The bottom panel of Figure 4 shows the background-subtracted data along with the bestfit sine wave, where the oscillation amplitude is $284 \mathrm{~km}$ and the period $154 \mathrm{~s}$. Comparing 


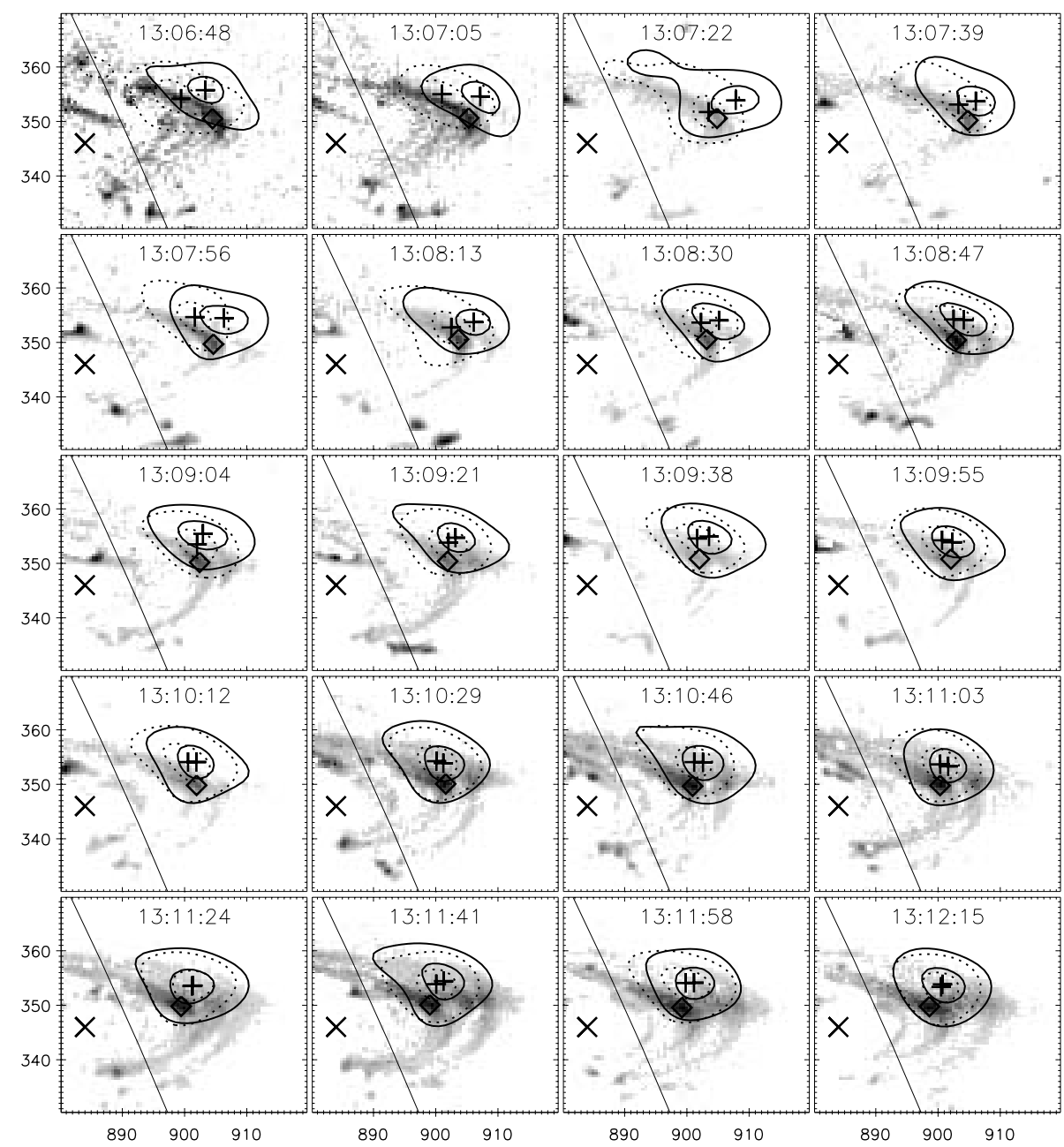

Figure 3. Time variations of the running difference images of TRACE $195 \AA$ (b-w reversed), overlaid on RHESSI contours at $60 \%$ and $90 \%$ of the peak intensity for $6-12$ (dashed line) and $12-25 \mathrm{keV}$ (solid line) images.

with the existed observations of coronal loop oscillations (see the book by Achwanden 2004), it seems that the oscillation in Figure 4 is a new phenomenon. First, the oscillation occurs during the shrinkage process. Second, the oscillatory loops are with the flare temperature and are the flare heated reconnection loops. Third, the oscillation is vertical rather than transverse.

We make a comparison of our oscillatory shrinkage with the 2.5-dimensional magnetic reconnection simulation by Chen et al. (1999). From their simulations we noticed that there seem to have two kinds of shrinkage: one happens at the early time of $\mathrm{t}<10 \tau_{A}$; the other is after $10 \tau_{A}$. Although Chen et al.. (1999) did not make detailed presentations for the early shrinkage process, we found that the observed TRACE loop shrinkage in the early impulsive phase is similar to the simulation result, especially $10 \tau_{A}$, ranging from 3 to 6 minutes, is just consistent with the observations. Furthermore, we found from the figure 4 of Chen et al. (1999) an oscillation of the looptop when it rises upward later. This calculated oscillatory expansion of the flare loop is similar to our observed oscillatory shrinkage of the flare loop, although one is overlapped on the upward motion, the other 

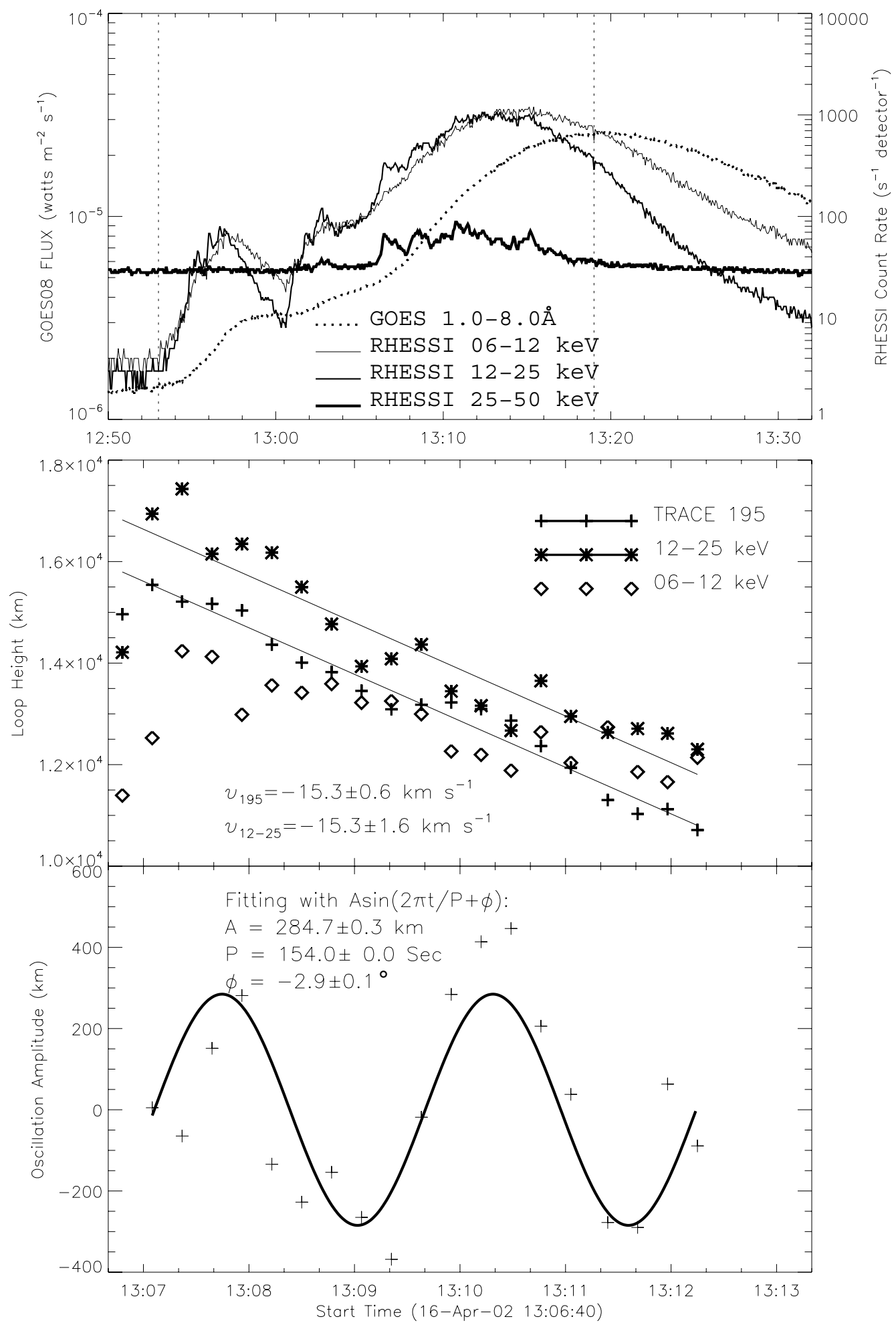

Figure 4. Top panel: GOES-08 SXR and RHESSI HXR light curves. The two vertical dotted lines mark the times of the beginning and maximum of the flare in 1-8 A. Middle panel: Time histories of the loop height obtained from 6-12 keV and 12-25 keV RHESI images as well as from TRACE $195 \AA$ Amages. The solid lines are the linear fits. Bottom panel: The TRACE loop height subtracted linear trend along with the best fit sine wave. 
is overlapped on the downward motion. We think that the explanation by Chen et al. (1999) for the oscillatory expansion is also suitable for the observed oscillatory shrinkage, i.e., the oscillation of reconnection rate itself, due to the imbalance between the inflow and outflow at the reconnection site, is the reason leading to the observed oscillatory shrinkage.

\section{Conclusions}

Based on RHESSI observations as well as associated TRACE observations, it seems to be clear at present about the morphology of flare X-ray sources and their motions. Especially, RHESSI observed a new kind of phenomenon: the downward motion of the looptop $\mathrm{X}$-ray source, or the shrinkage of flare loops at the early impulsive phase. The significance of this discovery in respect to the reconnection model is still not clear at present. Further studies are much necessary, so that the observations can be better understood.

\section{Acknowledgements}

This work is supported by the National Natural Science Foundation of China via grants 10333040 and 10221001.

\section{References}

Aschwanden, M. J. 2004, in: P.H. Rabinovich (ed.), Physics of the Solar Corona. An Introduction (Praxis Publishing Ltd., Chichester, UK, and Springer-Verlag Berlin)

Bogachev S. A. et al. 2005, ApJ 630, 572

Chen, P. F., Fang, C., Tang, Y. H., \& Ding, M. D. 1999, ApJ 513, 516

Dennis B. R. et al. 2005, in Proceedings of CESRA 2004, in press

Gan, W. Q. et al. 2004, in Proceedings IAU Symposium No. 223, p. 623

Gan, W. Q. 2006, in Proceedings of the 9th Asian-Pacific Regional IAU Meeting, p. 9

Grigis P. C. \& Benz A. O. 2005, ApJ (Letters) 625, L143

Krucker, S., Hurford, G. J., \& Lin, R. P. 2003, ApJ (Letters) 595, L103

Krucker S. \& Hudson H. S. 2004, in Proceedings of the SOHO 15 Workshop (ESA SP-575), p. 247

Li Y. P. \& Gan W. Q. 2005a, ChAA 29, 61

Li, Y. P., \& Gan, W. Q. 2005b, ApJ (Letters) 629, L137

Li, Y. P., \& Gan, W. Q. 2006, ApJ (Letters), submitted

Lin R. P. et al. 2002, Solar Phys. 210, 3

Lin R. P. et al. 2003, ApJ (Letters) 295, L89

Lin R. P. 2004, in Frontiers of Cosmic Ray Science, p. 335

Liu, W., Jiang, Y. W., Liu, S., \& Petrosian, V. 2004, ApJ (Letters) 611, L43

Masuda, S., Kosugi, T., Hara, H., Tsuneta, S., Ogawara, Y. 1994, Nature 371, 495

Priest, E. R., \& Forbes, T. G. 2002, Astron. Astrophys. Rev. 10, 313

Share G. H. \& Murphy R. J. 2004, IAUS 219, 133

Share G. H. \& Murphy R. J. 2005, AGU Monograph, Chapman Conference 2004, Solar Eruptions and Energetic Particles, ed. N. Gopalswamy, in press

Smith D. F. 2004, astro-ph/0404594

Sui, L., \& Holman, G. D. 2003, ApJ (Letters) 596, L251

Sui, L., Holman, G. D., \& Dennis, B. R. 2004, ApJ 612, 546

Tsuneta S. et al. 1992, PASJ 44, L63

Veronig A. M. et al. 2004, ApJ (Letters) 603, L117

Veronig, A. M., Karlický, M., Vršnak, B., Temmer, M., Magdalenić, J., Dennis, B. R., Otruba, W., \& Pözi, W. 2006, A\&̈A 446, 675 